\title{
A STRENGTHENED SCHWARZ-PICK INEQUALITY FOR DERIVATIVES OF THE HYPERBOLIC METRIC*
}

\author{
WENFA YUAN, DONGLI CHEN AND PINGAN WANG
}

\begin{abstract}
This paper is to investigate the Schwarz-Pick inequality for the hyperbolic derivative. Our result is not only a contraction but also a contraction minus a positive constant and this improves Beardon's theorem greatly.
\end{abstract}

\section{Introduction}

In the open unit disk $D \subset C$ (where $C$ is the complex plane), the hyperbolic metric $\rho$ is defined by

$$
\rho(z, w)=\frac{1}{2} \log \frac{1+\left|\varphi_{w}(z)\right|}{1-\left|\varphi_{w}(z)\right|}
$$

where $\tau \in D, \varphi_{\tau} \in \operatorname{Aut}(D)$, Aut $(D)$ denotes the automorphism on $D$ given by

$$
\varphi_{\tau}(\lambda)=\frac{\tau-\lambda}{1-\bar{\tau} \lambda}
$$

The Schwarz-Pick Lemma says that any analytic function $f: D \rightarrow D$ is nonincreasing under $\rho$, equivalently

$$
\rho(f(z), f(w)) \leq \rho(z, w), \quad \forall z, w \in D .
$$

Mercer $^{[4],[5]}$ proved a strengthened Schwarz-Pick inequality:

Lemma 1. Let $f: D \rightarrow D$ be analytic and $\tau \in D$. Then

$$
\rho(f(z), f(w)) \leq \rho(z, w)-B, \quad(B \geq 0)
$$

i.e.

$$
\rho(f(z), f(w)) \leq \rho(z, w)+\frac{1}{2} \log \left[1-(1-A) \frac{2\left|\varphi_{z}(w)\right|}{\left(1+\left|\varphi_{z}(w)\right|\right)^{2}}\right], \quad \forall z, w \in D
$$

Received November 22, 2004; revised March 11, 2005.

2000 Mathematics Subject Classification. 30C10.

Key words and phrases. Hyperbolic metric, derivative, analytic functions, Schwarz-Pick inequality.

Supported by the Special Science Foundation of the Educational Committee of Shaanxi Province (03JK065). 
where

$$
B=-\frac{1}{2} \log \left[1-(1-A) \frac{2\left|\varphi_{z}(w)\right|}{\left(1+\left|\varphi_{z}(w)\right|\right)^{2}}\right]
$$

and

$$
A=\left\{\begin{array}{cl}
\frac{\alpha+\left|\varphi_{\tau}(w)\right|}{1+\alpha\left|\varphi_{\tau}(w)\right|} & \text { if } \rho(z, \tau) \leq \rho(z, w) \\
\frac{\alpha+\left|\varphi_{\tau}(z)\right|}{1-\alpha\left|\varphi_{\tau}(z)\right|} & \text { if } \rho(\tau, w) \leq \rho(z, w) \\
\frac{\left(\left|\varphi_{w}(z)\right|-\alpha\right)\left(u^{2}+1\right)+2 u\left(\alpha\left|\varphi_{w}(z)\right|-1\right)}{2 u\left(\varphi_{w}(z)-\alpha\right)+\left(\alpha \varphi_{w}(z)-1\right)\left(u^{2}+1\right)} & \text { otherwise }\left(u=\max \left\{\left|\varphi_{\tau}(z)\right|,\left|\varphi_{\tau}(w)\right|\right\}\right)
\end{array}\right.
$$

Note that $A=\varphi_{\alpha}\left(-2 u /\left(u^{2}+1\right)\right)$ in the last line and hence $0 \leq A \leq 1$ in all cases, where $a=\varphi_{\alpha}\left(\left|\varphi_{w}(z)\right|\right)$.

Dieudone ${ }^{[3]}$ proved the following result:

Lemma 2. If $f: D \rightarrow D$ is analytic and $f(0)=0$, then

$$
\left|f^{\prime}(z)\right| \leq \begin{cases}1 & \text { if }|z| \leq \sqrt{2}-1 \\ \frac{\left(1+|z|^{2}\right)^{2}}{4|z|\left(1-|z|^{2}\right)} & \text { if }|z|>\sqrt{2}-1\end{cases}
$$

Later he pointd out that the above inequality, i.e., the so-called Schwarz Lemma for the derivative of $f$ is best possible for each value of $z \in D$.

The dick $D$ is endowed with the hyperbolic metric $d s^{*}=2 d z /\left(1-|z|^{2}\right)$, and the hyperbolic derivative $f^{*}(z)$ of $f$ at $z$ is given by

$$
f^{*}(z)=\left(\frac{1-|z|^{2}}{1-|f(z)|^{2}}\right) f^{\prime}(z) \text {. }
$$

Beardon $^{[1],[2]}$ obtained a Schwarz-Pick Lemma for derivatives, i.e.

Lemma 3. If $f: D \rightarrow D$ is analytic but not a conformal automorphism of $D$ with $f(0)=0$, then

$$
\rho\left(f^{*}(0), f^{*}(z)\right) \leq 2 \rho(0, z) .
$$

Furthermore, "=" holds for each $z$ if $f(z)=z^{2}$.

Below we use Mercer's result to improve Beardon's. 


\section{Main Result and Its Proof}

Let $w=0$, then from (2) and (3), we get

$$
B_{0}=-\frac{1}{2} \log \left[1-\left(1-A_{0}\right) \frac{2|z|}{(1+|z|)^{2}}\right]
$$

where

$$
A_{0}= \begin{cases}\frac{\alpha+|\tau|}{1+\alpha|\tau|} & \text { if } \rho(z, \tau) \leq \rho(z, 0) \\ \frac{\alpha+\left|\varphi_{\tau}(z)\right|}{1+\alpha\left|\varphi_{\tau}(z)\right|} & \text { if } \rho(\tau, 0) \leq \rho(z, 0) \\ \frac{(|z|-\alpha)\left(u^{2}+1\right)+2 u(\alpha|z|-1)}{2 u(|z|-\alpha)+(\alpha|z|-1)\left(u^{2}+1\right)} & \text { otherwise } u=\max \left\{\left|\varphi_{\tau}(z)\right|,|\tau|\right\} .\end{cases}
$$

We have as a consequence:

Theorem. If $f: D \rightarrow D$ is analytic but not a conformal automorphism of $D$ with $f(0)=0, z \in D$, then

$$
\rho\left(f^{*}(0), f^{*}(z)\right) \leq 2 \rho(0, z)-2 B_{0},
$$

where $B_{0}$ and $A_{0}$ are defined as in (5) and (6), and $f^{*}(z)=\frac{1-|z|^{2}}{1-|f(z)|^{2}} \cdot f^{\prime}(z)$ is the hyperbolic derivative. Furthermore, "=" holds for each $z$ if $f(z)=z^{2}$.

We begin with a preliminary Lemma:

Lemma 4. Let $z_{0}, w_{0} \in D$ and $\left|w_{0}\right|<\left|z_{0}\right|$. If $f: D \rightarrow D$ is analytic with $f(0)=0$, $f\left(z_{0}\right)=w_{0}$, then both $f^{*}(0)$ and $f^{*}\left(z_{0}\right)$ lie in the closed hypertolic disc $D=\left\{z \mid \rho\left(z, w_{0} / z_{0}\right)\right.$ $\left.\leq \rho\left(0, z_{0}\right)-B_{0}\right\}$.

Proof. As in [1], we are given $z_{0}$ and $w_{0}$ in $D$, so we define maps $h: D \rightarrow D$ and $g: D \rightarrow D$ by

$$
h=\frac{f(z)}{z}, \quad \frac{f(z)-f\left(z_{0}\right)}{1-f(z) \cdot \overline{f\left(\bar{z}_{0}\right)}}=g(z)\left(\frac{z-z_{0}}{1-\bar{z}_{0} z}\right),
$$

Then

$$
h(0)=f^{\prime}(0)=f^{*}(0), \quad h\left(z_{0}\right)=\frac{w_{0}}{z_{0}}, \quad g(0)=\frac{w_{0}}{z_{0}}, \quad g\left(z_{0}\right)=f^{*}\left(z_{0}\right) .
$$

Using (1) and (5), then we get

$$
\begin{aligned}
& \rho\left(f^{*}(0), w_{0} / z_{0}\right)=\rho\left(h(0), h\left(z_{0}\right)\right) \leq \rho\left(0, z_{0}\right)-B_{0} \\
& \rho\left(f^{*}\left(z_{0}\right), w_{0} / z_{0}\right)=\rho\left(g(0), g\left(z_{0}\right)\right) \leq \rho\left(0, z_{0}\right)-B_{0}
\end{aligned}
$$

This completes the proof of Lemma 4 . 
Proof of Theorem.

From the Lemma, we have

$$
\begin{aligned}
\rho\left(f^{*}(0), f^{*}\left(z_{0}\right)\right) & \leq \rho\left(f^{*}(0), \frac{w_{0}}{z_{0}}\right)+\rho\left(f^{*}\left(z_{0}\right), \frac{w_{0}}{z_{0}}\right) \leq \rho\left(0, z_{0}\right)-B_{0}+\rho\left(0, z_{0}\right)-B_{0} \\
& =2 \rho\left(0, z_{0}\right)-2 B_{0},
\end{aligned}
$$

where $B_{0}$ and $A_{0}$ are given by (5) and (6) respectively. If $f(z)=z^{2}$, then $f^{*}(0)=0$, $f^{*}(z)=\frac{2 z}{1+|z|^{2}}$, and $B_{0}=0$. Therefore $\rho(0, z)=\log \frac{1+|z|}{1-|z|}$ and this completes the proof.

\section{References}

[1] A. F. Beardon. The Schwarz-Pick lemma for derivatives, Pro. Amer. Math. Soc. 125 (1997), 3255-3256.

[2] A. F. Beardon and T. K. Came. A strengthening of the Schwarz-Pick inequality, Amer. Math. Monthly 99 (1992), 216-217.

[3] J. Dieudone. Recherches sur quelques problem relatifs aux polynomes et aux functions bornees d'une variable complexe, Ann. Sci. Ecole Norm. Sup. 48 (1931), 247-358.

[4] P. R. Mercer. On a strengthened Schwarz-Pick inequality, J. Math. Anal. and Appl. 234 (1999), 735-759.

[5] P. R. Mercer. Sharpened versions of the Schwarz lemma, J. Math. Anal. and Appl. 205 (1997), 508-511.

College of Science, Xi'an University of Architecture \& Technology, Xi'an Shaanxi 710055, P. R. China. 